\title{
ANALYSIS
}

\section{Least-cost tradeable risk permit scheme for controlling risk of introducing invasive alien species by shipping ${ }^{\text {ts }}$}

\author{
Hong Li Feng ${ }^{a, 1}$, David A. Hennessy ${ }^{b, *}$ \\ ${ }^{a}$ Department of Economics \& CARD, 560D Heady Hall, Iowa State University, Ames, IA 50011-1070, United States \\ ${ }^{b}$ Department of Economics \& CARD, 578C Heady Hall, Iowa State University, Ames, IA 50011-1070, United States
}

\section{A R T I C L E I N F O}

\section{Article history:}

Received 1 September 2007

Received in revised form 14 May 2008

Accepted 16 May 2008

Available online 21 June 2008

Keywords:

Biological control

Independence

Linearize

Permit trading

Risk abatement

Uniformly mixed pollutant

Weakest link

JEL classification:

Q25; H23; D62

\begin{abstract}
A B S T R A C T
The probability of alien species invasion is reasonably characterized by a weakest link technology. A tradeable risk permit system for shippers has been proposed as a means of efficiently reducing this invasion probability. Economic jointness arising from statistical independence among private risks for the weakest link technology implies that the risk instrument to be traded should be linear in the log of firm success probabilities, rather than linear in firm success probabilities.
\end{abstract}

(c) 2008 Elsevier B.V. All rights reserved.

\section{Introduction}

Alien species have undoubtedly caused great ecological and economic harm, even if costs are difficult to estimate (Pimentel et al., 2000; Pimentel et al., 2005; Hoagland and Jin, 2006; Kalogirou et al., 2007). International trade has been identified as an important factor in the introduction of alien species (Westphal et al., 2008). A variety of policy prescriptions have been proposed, including ambient taxes (Jones and Corona, 2008) and biosecurity effort subsidies (Horan and Lupi, 2005a).
A case of particular interest has been the role of commercial shipping as a transport medium for invasion of the North American Great Lakes. A very interesting and relevant analysis was provided in Horan and Lupi (2005b), who model the use of a risk trading permit scheme for shippers. In it, shipping firms take risk abatement actions and have the risk they present measured by a regulator. The regulator also imposes a certain overall risk constraint, and shipping firms trade permits for assessed risk such that the overall risk constraint is satisfied. Risks are independent and their risk constraint is linear in firm success probabilities.

\footnotetext{
is Senior authorship is not assigned.

* Corresponding author. Tel.: +1 5152946171.

E-mail addresses: hfeng@iastate.edu (H.L. Feng), hennessy@iastate.edu (D.A. Hennessy).

1 Tel.: +1 5152946307 .
} 
Relative to a linear risk constraint, herein we show that further cost efficiency gains can be obtained from exploiting linearity of the log of the overall success probability in the log of firm success probabilities. Then permits can be traded on a one-for-one basis such that, importantly, the aggregate likelihood of an invasion is preserved at a given level. One-for-one trading is important in that it avoids the need for assessing transfer coefficients, and so reduces the extent of transactions costs. In turn such costs can reduce trade liquidity, even to the point where the market is not viable.

\section{Algebra of invasion constraint}

The set of shipping firms is denoted by $i \in\{1, \ldots, n\} \equiv \Omega_{n}$, and each firm travels the same route just once (round trip) per year. The probability that the ith firm is responsible, at least in part, for an alien species invasion is specified as $q_{i}$ so that the probability of that firm's success in not being responsible for an invasion is $\bar{q}_{i} \equiv 1-q_{i}$. We refer to $q_{i}$ and $\bar{q}_{i}$ as, respectively, the individual failure and success probabilities. Under risk independence, the overall probability of an invasion is

$P\left(\overline{\mathrm{q}}_{1}, \ldots, \overline{\mathrm{q}}_{n}\right)=1-\prod_{i=1}^{n} \overline{\mathrm{q}}_{i}$

and the aggregate success probability is $\Pi_{i=1}^{n} \bar{q}_{i}=1-P\left(\bar{q}_{1}, \ldots, \bar{q}_{n}\right)$. This is a weakest link technology in that failure by any one firm leads to overall failure. Were all firms to pose the same risk, then we could write $\bar{q}_{i}=\hat{q}$ so that $P\left(\bar{q}_{1}, \ldots, \bar{q}_{n}\right)=1-\hat{q}^{n}$. Denote the probability sum as nq.

However, suppose $\hat{q}$ and $\delta$ are appropriately chosen such that firm 1 had success probability $\hat{q}+\delta$, firm 2 had success probability $\hat{q}-\delta$, and remaining firms continued to have success probability $\bar{q}_{i}=\hat{q}$. The probability sum remains $(n-2)$ $\hat{q}+(\hat{q}+\delta)+(\hat{q}-\delta)=n \hat{q}$. Then the overall probability of invasion becomes $P\left(\bar{q}_{1}, \ldots, \bar{q}_{n}\right)=1-\hat{q}^{n-2}(\hat{q}+\delta)(\hat{q}-\delta)=1-\hat{q}^{n}+\hat{q}^{n-2} \delta^{2} \geq 1-\hat{q}^{n}$, with strict inequality whenever $\delta>0$. Indeed, it is readily shown that the inequality applies over the simplex $S=$ $\left\{\left(\bar{q}_{1}, \ldots, \bar{q}_{n}\right): \bar{q}_{i} \geq 0 \forall i \in \Omega_{n}, \sum_{i=1}^{n} \bar{q}_{i}=n \hat{q}\right\}$, i.e. $P\left(\bar{q}_{1}, \ldots \bar{q}_{n}\right) \geq 1-\hat{q}^{n} \forall$ $\left(\overline{\mathrm{q}}_{1}, \ldots \overline{\mathrm{q}}_{n}\right) \in \mathrm{S}$ with strict inequality whenever coordinate values are not common ${ }^{2}$. This means that an increase in the dispersion of individual success probabilities, subject to the sum constraint $\sum_{i=1}^{n} \bar{q}_{i}=n \hat{q}$, decreases the overall success probability. The weakest link technology ensures that firms with a comparatively low individual success probability do harm to the overall success probability that is difficult to compensate for by the existence of firms with a comparatively high individual success probability.

As an illustration, Horan and Lupi (2005b) report that about 500 round trips take place in the Great Lakes region by transoceanic vessels. Over a 20 year time frame, suppose that

\footnotetext{
${ }^{2}$ There is a large literature on the theory of permutation symmetric functions such as $P\left(\bar{q}_{1}, \ldots, \bar{q}_{n}\right)$ on a simplex domain such as $S$. Inequality $P\left(\bar{q}_{1}, \ldots, \bar{q}_{n}\right) \geq 1-\hat{q}^{n}$ is an example of a majorization relation, a set of tools widely encountered in the theory of measuring species diversity (Marshall and Olkin, 1979; Tong, 1983).
}

$n=500(20)=10,000$ and $n \hat{q}=10,000 \hat{q}=9998$ so that $\hat{q}=0.9998$. If all $\overline{\mathrm{q}}_{i}$ are equal then the probability of invasion is $1-$ $(0.9998)^{10,000} \approx 0.86469$. If $\overline{\mathrm{q}}_{i}=1 \forall i \in\{1, \ldots, 499\}$ for each of these 20 years and $\bar{q}_{500}=0.9$, then the probability of invasion is $1-$ $(1)^{499 \times 20}(0.9)^{20}=0.87842$. The invasion probability is somewhat larger if the individual failure probability is concentrated in one firm when compared with equal dispersion across all firms.

In general, suppose that $n \hat{q}=n-\alpha$ with $0 \leq \alpha \leq 1$. If all $\bar{q}_{i}$ values are equal at $\hat{q}=1-\alpha / n$ then the probability of invasion by year $t$ is $P\left(\bar{q}_{1}, \ldots, \bar{q}_{n}\right)=1-(1-\alpha / n)^{n t}$, with

$\operatorname{Lim}_{n \rightarrow \infty} 1-\left(1-\frac{\alpha}{n}\right)^{n t}=1-e^{-\alpha t}$

A first-order Taylor series approximation around $\alpha \mathrm{t}=0$ yields $1-e^{-\alpha t} \approx 1-(1-\alpha \mathrm{t})=\alpha$. Alternatively, if $\overline{\mathrm{q}}_{1}=1-\alpha$ and $\overline{\mathrm{q}}_{i}=$ $1 \forall i \in\{2,3, \ldots, n\}$ then $\operatorname{Lim}_{n \rightarrow \infty} P\left(\bar{q}_{1}, \bar{q}_{2}=1, \ldots, \bar{q}_{n}=1\right)=1-(1-\alpha)^{t}(1)^{(n-1) t}$ $\approx \alpha$. So, as with the numerical illustration above, when $\alpha$ is in the neighborhood of zero then the discrepancy should be small whereas when at is in the neighborhood of 1 then the discrepancy can be large. In that case, for $\alpha=t=1$ then $1-e^{-\alpha t}$ $\approx 0.6321$ while $1-(1-\alpha)^{t}(1)^{(n-1) t}=1-(1-\alpha)=1$.

\section{Linear constraint}

In this section we will first lay out what is essentially the trading problem in Horan and Lupi (2005b). We will then present alternative specifications of the social planner's problem. The optimality conditions for these problems will then be contrasted to show, as Horan and Lupi (2005b) did, that a linear risk constraint is generally inefficient. Throughout we assume there is just one invasive species of concern.

Trading is likely to be in maximum allowable failure probabilities or maximum allowable levels of observable activities that are associated with failure, rather than in success probabilities. For this reason the analysis below will work with $q_{i}$ and not with individual success probability $\overline{q_{i}}$. The trading problem laid out in Eq. (5) of Horan and Lupi (2005b) may be characterized as follows. Pollution control costs are $c_{i}\left(q_{i}\right)$, a (likely distinct) decreasing and convex function for the ith firm. Each vessel owner chooses its own failure probability $q_{i}$, a probability level correctly discerned by the monitoring regulator. The firm receives a failure probability allowance endowment of $q_{i}^{0}$ and the equilibrium market price of a unit of the risk instrument is $\rho$. Before trading takes place, the social planner chooses the total permit number $Q$, such that $\sum_{i=1}^{n} q_{i}^{0}=Q$ so that the instrument's market price depends on $Q$, or $\rho=\rho(Q)$.

Assuming interior solutions and that the risk levels bind, then the firm's problem is to

$\operatorname{Min}_{q_{i}} c_{i}\left(q_{i}\right)+\rho \times\left(q_{i}-q_{i}^{0}\right) \quad$ s.t. $q_{i} \leq q_{i}^{0}$.

This is consistent with minimizing the firms' joint costs subject to the total permit constraint, i.e.,

$\operatorname{Min}_{\left(q_{1}, \ldots, q_{n}\right)} \sum_{i=1}^{n} c_{i}\left(q_{i}\right)+\rho \times\left(\sum_{i=1}^{n} q_{i}-Q\right)$. 
The first-order conditions for Eqs. (3) and (4) are

$\frac{\mathrm{d} c_{i}\left(q_{i}\right)}{\mathrm{d} q_{i}}=-\rho(\mathrm{Q}) \quad \forall i \in \Omega_{n}$,

where interior solutions are assumed. Write $q_{i}^{l}=g_{i}[\rho(Q)]$ as the market clearing solution to this optimality condition, where superscript $l$ is to identify the linear nature of the constraint in Eq. (4). The final step in the Horan and Lupi linear framework is to optimize over $Q$.

True social cost will depend on the invasion probability rather than on the sum of the failure probability allowance endowments, Q. Thus, rather than problem (4), the constrained social objective is to minimize the cost of protecting against invasion subject to a given probability of invasion. Labeling $\Phi$ as the invasion probability, the social cost minimization problem may be written as

$$
\begin{aligned}
& \operatorname{Min}_{\left(q_{1}, \ldots, q_{n}\right)} \sum_{i=1}^{n} c_{i}\left(q_{i}\right) \text { subject to } P\left(\bar{q}_{1}, \ldots, \bar{q}_{n}\right)=\Phi \\
& =\operatorname{Min}_{\left(q_{1}, \ldots, q_{n}\right)} \sum_{i=1}^{n} c_{i}\left(q_{i}\right) \text { subject to } \prod_{i=1}^{n}\left(1-q_{i}\right)=1-\Phi \\
& =\operatorname{Min}_{\left(q_{1}, \ldots, q_{n}\right)} \sum_{i=1}^{n} c_{i}\left(q_{i}\right) \text { subject to } \sum_{i=1}^{n} \operatorname{Ln}\left(1-q_{i}\right)=\operatorname{Ln}(1-\Phi) \\
& =\operatorname{Min}_{\left(q_{1}, \ldots, q_{n}\right)} \sum_{i=1}^{n} c_{i}\left(q_{i}\right)+\lambda \times\left[\operatorname{Ln}(1-\Phi)-\sum_{i=1}^{n} \operatorname{Ln}\left(1-q_{i}\right)\right],
\end{aligned}
$$

where $\lambda$ is the constraint Lagrangian multiplier and shadow value of a unit increase in $\operatorname{Ln}(1-\Phi)$. Of course $\lambda$ depends on $\Phi$, or $\lambda=\lambda(\Phi)$. The first-order conditions are

$$
\left(1-q_{i}\right) \frac{d c_{i}\left(q_{i}\right)}{\mathrm{d} q_{i}}=-\lambda(\Phi) \quad \forall \mathrm{i} \in \Omega_{n},
$$

with solutions $q_{i}^{m}$. Here, superscript $m$ is to identify the multiplicative nature of the constraint representing the overall probability of success in avoiding an invasion, i.e. $\Pi_{i=1}^{n}\left(1-q_{i}\right)=1-\Phi$ is multiplicative in individual success probabilities.

If permit trading, as represented by Eqs. (3) and (4), is to achieve the least-cost conditional on the given invasion probability $\Phi$, then Eqs. (5) and (7) should deliver the same solution values. Equating these optimality conditions requires $q_{i}^{m}=q_{i}^{l}=1-\lambda / \rho$, i.e., that firms make common choices if the market trading problem is to be socially optimal ${ }^{3}$. A common value for socially optimal individual success probabilities is unlikely since the only conditions on firm cost functions are that they be increasing and convex. In addition, given that the probability of failure is equal to $1-\Pi_{i=1}^{n}\left(1-q_{i}\right)$, satisfying $\sum_{i=0}^{n} q_{i}^{0}=Q$ for any given value of $Q$ does not guarantee that the targeted failure probability will be met.

\section{Incentives}

Given problems with trading under the linear risk constraint, Horan and Lupi turned to second-best trading programs. Instead, we ask how one might implement a first-best trading program. Can a trading mechanism be constructed that does

\footnotetext{
${ }^{3}$ Notice that the left hand side of Eq. (7) is an increasing function of $q$, so convexity is not a concern.
}

minimize cost subject to the invasion risk constraint? The answer is 'yes' and the transformation in Eq. (6) reveals this. Writing $z_{i} \equiv \operatorname{Ln}\left(1-q_{i}\right)$ then $1-q_{i}=e^{z_{i}}, c_{i}\left(q_{i}\right)=c_{i}\left(1-e^{z_{i}}\right) \equiv \widehat{c}_{i}\left(z_{i}\right)$ and the Lagrangian for the social welfare problem can be presented as

$\operatorname{Min}_{\left(z_{1}, \ldots, z_{n}\right)} \sum_{i=1}^{n} \widehat{C}_{i}\left(z_{i}\right)+\lambda \times\left[\operatorname{Ln}(1-\Phi)-\sum_{i=1}^{n} z_{i}\right]$.

There should not be any additional practical problems in trading log probabilities $z_{i}$ relative to probabilities as quantified by $q_{i}$ or $\bar{q}_{i}$; it is just a matter of getting used to how the instrument is presented for trading.

There remains the issue of specifying the instrument to be traded such that one buys permits to have a larger failure probability or take a riskier observable action, parallel to problem (3) above. Define $y_{i} \equiv-z_{i} \equiv-\operatorname{Ln}\left(1-q_{i}\right)$ so that $y_{i} \geq 0$. Also define $Y \equiv-\operatorname{Ln}(1-\Phi)$. Then, parallel to Eq. (3), the private optimization problem can be specified as

$\operatorname{Min}_{y_{i}} \widetilde{c}_{i}\left(y_{i}\right)+\lambda \times\left(y_{i}-y_{i}^{0}\right) \quad$ s.t. $y_{i} \leq y_{i}^{0}$.

where the firm's cost $\tilde{c}_{i}\left(y_{i}\right)$ is defined by $\tilde{c}_{i}\left(y_{i}\right) \equiv c_{i}\left(1-e^{-y_{i}}\right)$.

A permit market allowing one-for-one trading in $y_{i}$ minimizes the sum of costs associated with attaining the constraint that the log of the probability of no invasion is at least $\operatorname{Ln}(1-\Phi)$. This is because the log transformation has provided us with a problem that is isomorphic to that of permit trading for uniformly mixed pollutants, where an interior optimum requires marginal costs to be common (Montgomery, 1972). Linearization is possible under independent individual success probabilities. For a weakest link technology and independence among risks, the logs of individual success probabilities may be viewed as mixing uniformly to give the aggregate success probability. This single modification to the Horan and Lupi risk trading system brings the weight of the large literature on permit trading efficiency down in support of the novel and potentially important idea that they have developed.

\section{Conclusion}

Risk permit trading could conceivably have many applications beyond waterway access by transoceanic vessels. These might include limitations on the use of transgenetic species where again invasive species is the concern, and on the use of antibiotics where resistance is the concern. Inefficiencies from implementing a risk permit trading scheme that is linear in firm success probabilities rather than linear in the log of these probabilities are likely to be small or even negligible in many cases, but could be larger in other cases. If the linear risk sum constraint were thought to be more practical to implement, our analysis can be seen as a motive for restricting large positions in risk permits for any one vessel.

\section{R E F E R E N C E S}

Horan, R.D., Lupi, F., 2005a. Economic incentives for controlling trade-related biological invasions in the Great Lakes. Agricultural and Resource Economics Review 34, 75-89. 
Horan, R.D., Lupi, F., 2005b. Tradeable risk permits to prevent future introductions of invasive alien species into the Great Lakes. Ecological Economics 52, 289-304.

Hoagland, P., Jin, D., 2006. Science and economics in the management of an invasive species. Bioscience 56, 931-935.

Jones, K.R., Corona, J.P., 2008. An ambient approach to invasive species. Ecological Economics 64, 534-541.

Kalogirou, S., Corsini, M., Kondilatos, G., Wennhage, H., 2007. Diet of the invasive piscivorous fish Fistularia commersonii in a recently colonized are of the eastern Mediterranean. Biological Invasions 9, 887-896.

Marshall, A.W., Olkin, I., 1979. Inequalities: Theory of Majorization and Its Applications. Academic Press, San Diego.

Montgomery, W.D., 1972. Markets in licenses and efficient pollution control programs. Journal of Economic Theory 5 , 395-418.
Pimentel, D., Lach, L., Zuniga, R., Morrison, D., 2000.

Environmental and economic costs of nonindigenous species in the United States. Bioscience 50, 53-65.

Pimentel, D., Zuniga, R., Morrison, D., 2005. Update on the environmental and economic costs associated with alien-invasive species in the United States. Ecological Economics 52, 273-288.

Tong, Y.L., 1983. Some distribution properties of the sample species-diversity indices and their applications. Biometrics 39, 999-1008.

Westphal, M.I., Browne, M., MacKinnon, K., Noble, I., 2008. The link between international trade and the global distribution of invasive alien species. Biological Invasions 10, 391-398. 
lowa State University does not discriminate on the basis of race, color, age, religion, national origin, sexual orientation, gender identity, genetic information, sex, marital status, disability, or status as a U.S. veteran. Inquiries can be directed to the Director of Equal Opportunity and Compliance, 3280 Beardshear Hall, (515) 294-7612. 